hep-th/0612072

UG-06-09

IFT-UAM/CSIC-06-51

UB-ECM-PF-06-34

\title{
Seven-branes and Supersymmetry
}

\author{
Eric A. Bergshoeff $\diamond$, Jelle Hartong $\diamond$, Tomás Ortín ${ }^{\star}$ and Diederik Roest \\ $\diamond$ Centre for Theoretical Physics, University of Groningen, \\ Nijenborgh 4, 9747 AG Groningen, The Netherlands \\ E.A.Bergshoeff, J.Hartong@rug.nl \\ as Instituto de Física Teórica UAM/CSIC, \\ Facultad de Ciencias C-XVI, C.U. \\ Cantoblanco, E-28049-Madrid, Spain \\ Tomas.Ortin@cern.ch \\ ه Departament Estructura i Constituents de la Materia, \\ Facultat de Física, Universitat de Barcelona, \\ Diagonal, 647, 08028 Barcelona, Spain \\ droest@ecm.ub.es
}

\begin{abstract}
We re-investigate the construction of half-supersymmetric 7-brane solutions of IIB supergravity. Our method is based on the requirement of having globally well-defined Killing spinors and the inclusion of $S L(2, \mathbb{Z})$-invariant source terms. In addition to the well-known solutions going back to Greene, Shapere, Vafa and Yau we find new supersymmetric configurations, containing objects whose monodromies are not related to the monodromy of a D7-brane by an $S L(2, \mathbb{Z})$ transformation.
\end{abstract}




\section{Introduction}

The construction of half-supersymmetric 7-brane solutions goes back to the classic work [1] where these were presented as cosmic string solutions of a $D=4$ gravity plus dilatonaxion system. Later, after the invention of D-branes [2], these solutions were oxidized to $D=10$ dimensions and re-interpreted as D7-brane solutions [3]. Since then, D7-branes, in particular in the form of D3-D7-brane systems, have found important applications in model building, see e.g. [4-6], and cosmology, see e.g. [7-9].

The original motivation of [1] was not the construction of cosmic string solutions as such, but the investigation of supersymmetric String Theory backgrounds that are more general than the direct product of 4-dimensional Minkowski space-time and a Calabi-Yau 3 -fold. The construction of [1] assumes that the gravity plus dilaton-axion system arises from compactification on a torus $T^{2}$, the complex axidilaton field $\tau$ being its modular parameter. The cosmic string solutions found in [1] can be seen as products of a 2dimensional Minkowski space-time (the worldsheet of the cosmic string) and a nontrivial 4-dimensional space with the structure of a $T^{2}$ fibration over a 2-dimensional base space (the transverse space of the cosmic string). Supersymmetry requires this 4-dimensional space to be a Calabi-Yau 2-fold. Within the context of $D=10$ IIB supergravity one must for this purpose rely on a 12-dimensional F-theory [10].

It is the purpose of this work to re-analyze half-supersymmetric 7-brane solutions of IIB supergravity without invoking a higher-dimensional origin of the gravity plus axidilaton system. We will, instead, directly analyze the IIB supergravity Killing spinor equations, taking into account all their symmetries, and we will require that there exists a globally well-defined Killing spinor. We will find that this supersymmetry requirement is less restrictive than the one imposed in [1].

Another distinguishing feature of our analysis is that we add $S L(2, \mathbb{Z})$-invariant source terms to the equations of motion 1 . These source terms represent the coupling of a 7 -brane to the IIB supergravity background. They enable us to derive an expression for the 7brane solution in a neighborhood of the brane source as well as the monodromy of the fields around the brane source in terms of the brane source charges.

In general, D7-branes do not come alone since this leads to singularities at a finite distance from the D7-brane. To obtain a globally well-defined solution one can add other 7-brane objects whose monodromy is not related to the monodromy of a D7-brane by an $S L(2, \mathbb{Z})$-transformation 2 . We will call these objects, for reasons that will become clear soon, "det $Q>0$-branes". The existence of these new objects may be anticipated from the observation that the standard Ramond-Ramond $(\mathrm{RR})$ potential $C_{(8)}$ that couples to the D7-brane is part of a triplet $C_{(8)}^{\alpha \beta}$ of 8 -form potentials under $S L(2, \mathbb{Z})$, i.e.

$$
C_{(8)} \rightarrow C_{(8)}^{\alpha \beta}
$$

\footnotetext{
${ }^{1}$ Strictly speaking the source terms are $S L(2, \mathbb{Z})$-invariant provided we also transform the constants that occur in these source terms, see eq. (2.1).

${ }^{2}$ In this work we will also present an alternative way to cure the singularity by the addition of an $S L(2, \mathbb{Z})$-transformed D7-brane, see section 6.4.
} 
and that not each combination of potentials is related to $C_{(8)}$ via an $S L(2, \mathbb{Z})$-transformation.

The multiple D7-brane solutions of [1] can be viewed as special configurations where the $\operatorname{det} Q>0$-branes occur in particular groups such that their masses and monodromies cancel amongst each other and one is left with multiple D7-branes only. We will present new half-supersymmetric configurations where these cancellations do not occur and we will discuss the properties of these solutions. A distinguishing feature of these new solutions is that the metric has a deficit angle at the position of each $\operatorname{det} Q>0$-brane that is not cancelled. We will construct special solutions that can be used as the basic building blocks for constructing all multiple 7-brane solutions including the multiple D7-branes of [1].

It is not clear what the correct interpretation of the $\operatorname{det} Q>0$-branes is within String Theory. Part of this work's motivation was to show explicitly that within the context of supergravity one can allow for such objects. At present their world-volume dynamics is not well-understood.

This paper is organized as follows: In section 2 we will discuss the $S L(2, \mathbb{Z})$-invariant source terms. The analysis of the equations of motion, with emphasis on the existence of a Killing spinor, will be the subject of section 3. We will discuss a BPS equation for the 7brane solutions in section 4. Next, we will describe how to construct globally well--defined 7-brane solutions in section 5. Explicit examples of solutions, old ones and new ones, will be presented in section 6. Finally, we give our conclusions in section 7.

\section{Seven-brane Source Terms}

The gravity plus axidilaton system we are going to consider is a consistent truncation of the IIB supergravity action [13-15] in which only the metric, the RR 0-form (axion) $\chi$ and the dilaton $\phi$ are kept. These two scalar fields appear in the complex combination $\tau=\chi+i e^{-\phi}$ (the axidilaton), which parameterizes an $S L(2, \mathbb{R}) / S O(2)$ coset.

The coupling of a 7-brane, labelled by the real numbers $p, q, r$, to the gravity plus axidilaton system is described by the following Einstein-frame "pseudo action" 3 :

$$
\begin{aligned}
S= & \frac{g_{s}^{2}}{16 \pi G_{N}^{(10)}} \int d^{10} x \sqrt{-g}\left[R-\frac{\partial_{\mu} \tau \partial^{\mu} \bar{\tau}}{2(\operatorname{Im} \tau)^{2}}\right. \\
& \left.-\int_{\Sigma} d^{8} \sigma \sqrt{-g_{(8)}} \frac{\delta(x-X(\sigma))}{\sqrt{-g}} \frac{1}{\operatorname{Im} \tau}\left(p+q|\tau|^{2}+r \frac{\tau+\bar{\tau}}{2}\right)\right] .
\end{aligned}
$$

The 7 -brane world-volume, $\Sigma$, is parameterized in the above action by $\left\{\sigma^{i}, i=0,1, \ldots, 7\right\}$. The metric on the world-volume is $g_{(8) i j}$ which is the pull-back of the target-space Einsteinframe metric $g_{\mu \nu}$. The embedding coordinates of the brane are denoted by $X^{\mu}(\sigma)$, and so the pull-back is given by

\footnotetext{
${ }^{3}$ The reason that we call the action (2.1) a pseudo action will become clear shortly.
} 


$$
g_{(8) i j}(\sigma)=\frac{\partial X^{\mu}}{\partial \sigma^{i}} \frac{\partial X^{\nu}}{\partial \sigma^{j}} g_{\mu \nu}(X) .
$$

We are only considering objects for which in the static gauge the transverse scalars are set equal to zero, i.e. we do not consider fluctuations of the world-volume. The source term in (2.1) should be interpreted as adding a purely static object to the theory. Note that the source term is linear in $p, q$ and $r$. This is related to the fact that, unlike e.g. strings, all 7-branes have the same half-supersymmetry projection operator, see (3.8), which is invariant under $S L(2, \mathbb{R})$ transformations.

In the coefficient in front of the above action $g_{s}$ is the string coupling constant (i.e. the vacuum expectation value of $e^{\phi}$ measured at infinity) and $G_{N}^{(10)}$ is the 10-dimensional Newton constant which is given by

$$
G_{N}^{(10)}=8 \pi^{6} g_{s}^{2} \ell_{s}^{8}
$$

where $\ell_{s}$ is the string length $\sqrt{\alpha^{\prime}}$. Then, the coefficient in front of the action (2.1) is independent 4 of $g_{s}$. The relative numerical coefficient between the brane probe action and the bulk action is just 1 due to the coincidence:

$$
T_{D 7} g_{s}=\frac{g_{s}^{2}}{16 \pi G_{N}^{(10)}} .
$$

The bulk action is invariant under $S L(2, \mathbb{R})$ transformations which act on the axidilaton according to

$$
\tau \rightarrow \Lambda \tau \equiv \frac{a \tau+b}{c \tau+d} \quad \text { where } \quad \Lambda=\left(\begin{array}{ll}
a & b \\
c & d
\end{array}\right) \in S L(2, \mathbb{R})
$$

and leave the Einstein-frame metric $g_{\mu \nu}$ invariant. Observe that the coefficient in front of the action is $S L(2, \mathbb{R})$-invariant precisely because it does not depend on $g_{s}$.

The worldvolume term in the action is also $S L(2, \mathbb{R})$-invariant provided that the real constants $p, q, r$, arranged in the traceless matrix

$$
Q \equiv\left(\begin{array}{cc}
r / 2 & p \\
-q & -r / 2
\end{array}\right)
$$

transform in the adjoint representation of $S L(2, \mathbb{R})$. Note that the determinant of this matrix,

$$
\operatorname{det} Q=q p-r^{2} / 4
$$

is $S L(2, \mathbb{R})$-invariant and can be used as a label to distinguish between different conjugacy classes.

\footnotetext{
${ }^{4}$ One may recover the form of the action as it appears in low-energy perturbative string theory which has a factor $g_{s}^{-2}$ in front of it by going to the so-called modified Einstein-frame metric $\tilde{g}_{\mu \nu} \equiv g_{s}^{1 / 2} g_{\mu \nu}[16]$.
} 
It is well known that the classical invariance of this theory is broken by quantummechanical effects such as charge quantization to $S L(2, \mathbb{Z})$. Hence, from now on we will only consider this group. Observe that, actually, $\tau$ transforms only under the group $P S L(2, \mathbb{Z})=$ $S L(2, \mathbb{Z}) /\{ \pm \mathbb{1}\}$ since $-\mathbb{1}$ leaves it invariant.

The reader may notice that the source term present in the pseudo action (2.1) contains only a Nambu-Goto (NG) term and no Wess-Zumino (WZ) term. At first sight this seems surprising. For instance, in the case of the D7-brane, which corresponds to the case that $p=1$ and $q=r=0$ the source term contains only the dilaton and there is no source term for the axion whereas the D7-brane is known to have a magnetic axionic charge. The reason that we nevertheless will be able to reproduce the D7-brane solution is that we will only consider solutions for which the axidilaton $\tau$ is a holomorphic function of the two coordinates transverse to the D7-brane. This input comes from a consideration of the Killing spinor equations, see subsection (3.1). Since the dilaton and axion are combined in one holomorphic function it is enough to consider a source term for the dilaton only. The action (2.1) is only a convenient tool for investigating supersymmetric 7-brane solutions. That is the reason that we call it a pseudo action. For the derivation of a proper action and a justification for the use of action (2.1) we refer to [19].

$S L(2, \mathbb{Z})$-invariant 7 -brane world-volume actions were considered in [20] and were shown to preserve half of the supersymmetries for all possible values of $p, q, r$. The world-volume action describing a single D7-brane or any $S L(2, \mathbb{Z})$ transform thereof has values $p, q, r$ which satisfy the condition $-r^{2} / 4+p q=0$, or det $Q=0$. For this set of 7 -brane actions one can introduce a single Born-Infeld vector in a target-space gauge-invariant and $S L(2, \mathbb{Z})$ invariant manner [11]. This confirms the identification of these objects as Dirichlet branes or $S L(2, \mathbb{Z})$ transforms thereof. It turns out (see section 5 ) that in constructing globally well-defined solutions containing a D7-brane, objects with $\operatorname{det} Q>0$ play a crucial role while on the other hand the possibility $\operatorname{det} Q<0$ never arises. It is the purpose of this paper to find out more about the status of the $\operatorname{det} Q>0$ objects.

By a "D7-brane" we mean any representative element of the $\operatorname{det} Q=0 S L(2, \mathbb{R})$ conjugacy class 5 . This is because in constructing finite energy solutions we divide out type IIB supergravity by the duality group $S L(2, \mathbb{Z})$ (or a subgroup thereof). In doing so one can no longer distinguish the various elements of a particular conjugacy class where each conjugacy class is characterized by the value of $\operatorname{det} Q$.

\section{The Equations of Motion}

\subsection{Supersymmetry and holonomy of the Killing spinor}

We are considering supersymmetric solutions of the system (2.1) and we thus require that the following Killing spinor equations are satisfied (using the supersymmetry rules of [21]):

\footnotetext{
${ }^{5}$ The supergravity solutions in this article which describe the space-time close to a 7-brane are characterized by the value of $\operatorname{det} Q$. This value labels $S L(2, \mathbb{R})$ conjugacy classes. Whenever we speak of a conjugacy class we will always mean of $S L(2, \mathbb{R})$ and not of $S L(2, \mathbb{Z})$.
} 


$$
\begin{aligned}
\delta_{\epsilon} \lambda & =\frac{i}{\tau-\bar{\tau}}\left(\gamma^{\mu} \partial_{\mu} \bar{\tau}\right) \epsilon_{C}=0, \\
\delta_{\epsilon} \psi_{\mu} & =\left(\partial_{\mu}+\frac{1}{4} \omega_{\mu}^{a b} \gamma_{a b}+\frac{1}{4(\tau-\bar{\tau})} \partial_{\mu}(\tau+\bar{\tau})\right) \epsilon=0 .
\end{aligned}
$$

The Killing spinor $\epsilon$ can be written as $\epsilon=\epsilon_{1}+i \epsilon_{2}$ where $\epsilon_{1}$ and $\epsilon_{2}$ are two Majorana-Weyl spinors. The chirality of $\epsilon$ is negative, i.e. $\gamma_{11} \epsilon=-\epsilon$. The $C$ operation leaves Majorana spinors invariant. The equations (3.1) and (3.2) transform covariantly under the following $S L(2, \mathbb{Z})$ transformations

$$
\tau \rightarrow \frac{a \tau+b}{c \tau+d}, \quad \lambda \rightarrow e^{3 i \varphi} \lambda, \quad \psi_{\mu} \rightarrow e^{i \varphi} \psi_{\mu}, \quad \epsilon \rightarrow e^{i \varphi} \epsilon, \quad\left(\begin{array}{ll}
a & b \\
c & d
\end{array}\right) \in S L(2, \mathbb{Z})
$$

where $\varphi=\frac{1}{2} \arg (c \tau+d)$. This means that $\epsilon$ transforms under the double cover of $S L(2, \mathbb{Z})$. We define $-1=e^{i \pi}$ and $1=e^{i 0}$. There is no restriction on the range of $\varphi$. Numbers such as $e^{-i \pi}$ and $e^{2 i \pi}$ lie on another Riemann sheet.

The discrete group $S L(2, \mathbb{Z})$ is generated by the two elements $T$ and $S$, which are defined as follows:

$$
T=\left(\begin{array}{ll}
1 & 1 \\
0 & 1
\end{array}\right), \quad S=\left(\begin{array}{cc}
0 & -1 \\
1 & 0
\end{array}\right)
$$

Observe that, unlike $\tau$, the Killing spinor $\epsilon$ does transform under $S^{2}=-\mathbb{1}$ as $\epsilon \rightarrow i \epsilon$. Under $S^{4}=\mathbb{1}$ we have $\epsilon \rightarrow-\epsilon$. Only $S^{8}$ acts as the identity on $\epsilon$.

The transformation rules of the spinors tell us that they carry $U(1)$ charge. Actually, the term

$$
\mathcal{Q}_{\mu} \equiv \frac{1}{2 i} \frac{\partial_{\mu}(\tau+\bar{\tau})}{(\tau-\bar{\tau})},
$$

in the gravitino supersymmetry transformation rule (3.2) is a $U(1)$ connection and the whole operator that acts on $\epsilon$ is a $U(1)$ and Lorentz covariant derivative. In fact, the coset $S L(2, \mathbb{R}) / U(1)$ is a special Kähler manifold with Kähler potential $\mathcal{K}=\log \operatorname{Im} \tau$ and the above $U(1)$ connection is nothing but the pullback over the spacetime of the Kähler connection

$$
\mathcal{Q}=\frac{1}{2 i}\left(d \tau \partial_{\tau} \mathcal{K}-d \bar{\tau} \partial_{\bar{\tau}} \mathcal{K}\right)=\frac{1}{2 i} \frac{d(\tau+\bar{\tau})}{(\tau-\bar{\tau})} .
$$

Under isometries of the Kähler manifold (here the group $S L(2, \mathbb{R})$ ) the Kähler potential is only invariant up to Kähler transformations which become $U(1)$ transformations of the Kähler connection. This point will play a role in discussing under which condition the 
Killing spinors are well defined. We will also need the expression of the Kähler 2-form $\Omega$, which is the field strength of the Kähler connection:

$$
\Omega \equiv d \mathcal{Q}=\frac{1}{2 i} \frac{d \tau \wedge d \bar{\tau}}{(\operatorname{Im} \tau)^{2}}
$$

The $S L(2, \mathbb{Z})$-invariant supersymmetry projection operator of a 7 -brane extended in the directions $x^{1}, \cdots, x^{7}$ is given by

$$
P \epsilon=\frac{1}{2}\left(1-i \gamma_{\underline{0} \ldots \underline{7}}\right) \epsilon=\frac{1}{2}\left(1+i \gamma_{\underline{8}} \gamma_{\underline{9}}\right) \epsilon=0 .
$$

It follows that $\left(\gamma_{\underline{8}}+i \gamma_{\underline{9}}\right) \epsilon=0=\left(\gamma_{\underline{8}}-i \gamma_{9}\right) \epsilon_{C}$. If we assume that $\tau$ and the metric do not depend on the worldvolume coordinates $x^{0}, \cdots, x^{7}$ and choose a conformally-flat transverse metric, then Eq. (3.1) tells us that $\left(\partial_{8}-i \partial_{9}\right) \bar{\tau}=0$. We define the complex transverse coordinate $z=x^{8}+i x^{9}$ so that we now have $\partial_{z} \bar{\tau}=0$, that is, $\tau$ is a holomorphic function. In complex coordinates the condition on $\epsilon$ can be written as $\gamma_{\underline{z}^{*}} \epsilon=0$. Under these conditions, the most general 7-brane solution to equations (3.1) and (3.2) is given by $[3,22-24]$

$$
\begin{aligned}
d s^{2} & =-d t^{2}+d \vec{x}_{7}^{2}+(\operatorname{Im} \tau)|f|^{2} d z d \bar{z} \\
\tau & =\tau(z), \quad f=f(z), \\
\epsilon & =(f / \bar{f})^{1 / 4} \epsilon_{0},
\end{aligned}
$$

where $\epsilon_{0}$ is a constant spinor which satisfies $\gamma_{\underline{z}^{*}} \epsilon_{0}=0$. We will study in the next sections how these supersymmetric configurations solve the classical equations of motion corresponding to the action (2.1).

The functions $\tau$ and $f$ are assumed to be defined on the Riemann sphere. The form of the solution is therefore fixed up to $S L(2, \mathbb{C})$ transformations

$$
z \rightarrow \frac{a z+b}{c z+d}, \quad\left(\begin{array}{ll}
a & b \\
c & d
\end{array}\right) \in S L(2, \mathbb{C}) .
$$

These are the most general global coordinate transformations that do not change the structure of the branch cuts and singularities of $\tau$ and $f$ in the complex $z$-plane. Note that locally (but not globally) we can always choose a basis in which $f(z)=1$.

Although the configurations (3.9)-(3.11) are locally supersymmetric, they must satisfy further conditions to be globally well-defined and supersymmetric. The main issue here will be the possible multi-valuedness of $\tau(z)$ and $f(z)$, which in general will be holomorphic functions with singularities and branch cuts and which appear in the metric and Killing spinor.

The axidilaton $\tau$, being a physical field of IIB supergravity, must be single-valued. However, when constructing solutions, we will consider the IIB supergravity theory divided out 
by (a subgroup of $S L(2, \mathbb{Z}) 6$. Therefore, we consider values of $\tau$ related by transformations belonging to (a subgroup of) $P S L(2, \mathbb{Z})$ as equivalent. In particular, $\tau(z)$ may jump to $\Lambda \tau(z)$ when crossing a branch cut. In other words, it may have a non-trivial monodromy contained in (a subgroup of) $P S L(2, \mathbb{Z})$.

We will now derive the transformation rule for the function $f$ when going around a 7-brane by requiring that the holonomy of $\epsilon$ be well-defined. The holonomy of the Killing spinor is computed with respect to the generalized connection in (3.2), which is the sum of the Lorentz connection and $U(1)$ connection. The integrability condition of (3.2) requires that the total curvature vanishes but the Riemann curvature of the transverse space and the $U(1)$ curvature are, separately, non-trivial.

If we parallel-transport the Killing spinor $\epsilon$ using the connection in (3.2), evaluated on the solution (3.93.10) from a base point $b$ around a closed loop $\gamma_{b}$ it can be shown that the holonomy (with respect to the Lorentz group) of $\epsilon$ is given by

$$
\epsilon(b) \rightarrow \exp \left(\frac{i}{2} \operatorname{Im} \oint_{\gamma_{b}}(\log f)^{\prime} d z\right) \epsilon(b),
$$

where the prime denotes differentiation with respect to $z$.

The holonomy phase factor will depend on the base point $b$ but only on the homotopy class of $\gamma_{b}$ due to the vanishing total curvature. We require 7 it to be an $S L(2, \mathbb{Z})$ transformation as given in equation (3.3)

$$
\exp \left(\frac{i}{2} \operatorname{Im} \oint_{\gamma_{b}}(\log f)^{\prime} d z\right)=e^{i \varphi}
$$

such that the holonomy with respect to the generalized connection is trivial. Let $\gamma_{b}$ be parameterized by $\lambda \in[0,1]$. Then

$$
\exp \left(\frac{i}{2} \operatorname{Im} \oint_{\gamma_{b}}(\log f)^{\prime} d z\right)=\left(\frac{f(\lambda=1)}{|f(\lambda=1)|)}\right)^{1 / 2}\left(\frac{|f(\lambda=0)|}{f(\lambda=0))}\right)^{1 / 2}
$$

The requirement (3.14) then leads to the following condition for the function $f$

$$
f(\lambda=1)=(c \tau+d) f(\lambda=0) .
$$

Thus, when crossing a branch cut at the point $z$ we must have

$$
f(z) \rightarrow(c \tau(z)+d) f(z) .
$$

For the convenience of the reader we summarize some of the $S L(2, \mathbb{Z})$ properties of $\tau, f$ and $\epsilon$ in table 1 .

\footnotetext{
${ }^{6}$ In the case of dividing out by $S L(2, \mathbb{Z})$ this means that we are effectively dealing with a $64+64$ $N=1, D=8$ supergravity multiplet coupled to a $8+8$ vector multiplet instead of the $128+128$ IIB supergravity multiplet. Further, there are additional vector multiplets coming from the presence of 7 branes. The reduction is over the two directions transverse to the 7-branes and is triggered by the fact that $\tau$ is not an arbitrary holomorphic function of $z$.

${ }^{7}$ In general one can also allow for nontrivial spin structures but we will not do so here.
} 


\begin{tabular}{||c|c|c||}
\hline fields & group & order of $S$ \\
\hline \hline$\tau$ & $P S L(2, \mathbb{Z})$ & 2 \\
$f$ & $S L(2, \mathbb{Z})$ & 4 \\
$\epsilon$ & double cover & 8 \\
\hline
\end{tabular}

Table 1: Some $S L(2, \mathbb{Z})$ properties of $\tau, f$ and $\epsilon$.

The metric $g_{\mu \nu}$ is a physical field which must be single-valued modulo coordinate transformations. On the other hand, $\operatorname{Im} \tau$ appears explicitly in the expression (3.9) for $g_{\mu \nu}$ and it may transform into $|c \tau+d|^{-2} \operatorname{Im} \tau$ when crossing a branch cut. In general, the extra factor $|c \tau+d|^{-2}$ cannot be eliminated by an $S L(2, \mathbb{C})$ transformation and, thus, it must be compensated by $f(z)$. From (3.17) we see that the metric remains invariant when going around a 7-brane.

It is worth pausing a moment to compare the present situation with that of Ref. [30]. The system of Killing spinor equations studied there is essentially identical to the system studied here 8 . In particular, the gravitino supersymmetry transformation rule in [30] contains the Lorentz connection and a $U(1)$ connection which, on shell, is, up to gauge transformations, the Kähler connection of the scalar manifold to which a Chern-Simons supergravity is coupled. In fact, they find cosmic-string solutions that include those studied here and those found in [32] in $N=2, D=4$ theories with vector multiplets. The authors of [30], however, required the Killing spinors to have trivial monodromies up to signs corresponding to the non-trivial spin structure of the transverse space while here it is required that the monodromies should be trivial up to $U(1)$ transformations corresponding to the non-trivial $\operatorname{spin}^{c}$ structures of the transverse space. These are the right structures for $U(1)$-charged spinors and they allow for more general monodromies than those considered in $[30]$.

\subsection{The scalar equations of motion}

In this section we are going to study how the supersymmetric configurations found in the previous section solve the scalar equation of motion with sources derived from the action (2.1). We perform a variation of the action (2.1) with respect to $\bar{\tau}$ and use the metric (3.9). This leads to the following equation of motion for $\tau$ :

$$
\partial \bar{\partial} \tau-2 \frac{\partial \tau \bar{\partial} \tau}{\tau-\bar{\tau}}=-\frac{i}{4} \delta\left(z-z_{0}, \bar{z}-\bar{z}_{0}\right)\left(p+q \tau^{2}+r \tau\right)
$$

Due to the presence of the delta function 9 we cannot at this stage assume that $\tau$ is a

\footnotetext{
${ }^{8}$ The eight worldvolume dimensions of the 7-brane solutions do not play any role and we can view this system as, effectively, $2+1$-dimensional. We will do this to compute the mass in section 4.

${ }^{9}$ We define$$
\int \frac{i}{2} d z \wedge d \bar{z} \delta(z, \bar{z})=1
$$ 
globally holomorphic function. Equation (3.18) can be integrated as follows. Let $R$ be an infinitesimal disk $\left|z-z_{0}\right| \leq \delta$ and let us denote its boundary by $\gamma_{\delta}$. Integrating equation (3.18) over $R$ we obtain

$$
\lim _{\delta \rightarrow 0} \int_{R}\left(\partial \bar{\partial} \tau-2 \frac{\partial \tau \bar{\partial} \tau}{\tau-\bar{\tau}}\right) \frac{i}{2} d z \wedge d \bar{z}=-\frac{i}{4} \lim _{\delta \rightarrow 0} \oint_{\gamma_{\delta}} \tau^{\prime} d z=-\frac{i}{4}\left(p+q \tau^{2}+r \tau\right)_{z=z_{0}}
$$

where the prime denotes differentiation with respect to $z$. We have used Green's theorem 10 to relate the integral over $R$ to the integral over the boundary $\gamma_{\delta}$ and the fact that $\bar{\partial} \tau=0$ over $\gamma_{\delta}$.

Assuming that when $q, r \neq 0$ the $\operatorname{limit}_{z \rightarrow z_{0}} \tau$ exists one may write

$$
2 \pi i \tau\left(z_{0}\right)=\lim _{\delta \rightarrow 0} \oint_{\gamma_{\delta}} \frac{\tau}{z-z_{0}} d z
$$

Therefore we have

$$
\lim _{\delta \rightarrow 0} \oint_{\gamma_{\delta}}\left(2 \pi i \tau^{\prime}-p \frac{1}{z-z_{0}}-q \frac{\tau^{2}}{z-z_{0}}-r \frac{\tau}{z-z_{0}}\right) d z=0 .
$$

This form of the scalar equations of motion is convenient to derive an approximation of the possible solutions close to the source terms at $z_{0}$. This derivation goes as follows. We assume that the integrand of (3.21) is an analytic function without any poles in the interior of $\gamma_{\delta}$. Then it admits in $R$ a Taylor expansion

$$
2 \pi i \tau^{\prime}-p \frac{1}{z-z_{0}}-q \frac{\tau^{2}}{z-z_{0}}-r \frac{\tau}{z-z_{0}}=\sum_{n=0}^{\infty} a_{n}\left(z-z_{0}\right)^{n} .
$$

In the limit $\left|z-z_{0}\right| \rightarrow 0$ the poles on the left hand-side will dominate all the terms on the right hand-side. In this approximation the right hand-side of (3.22) can be put to zero, and we are left with the homogeneous version of equation (3.22), i.e.

$$
2 \pi i \tau^{\prime}-p \frac{1}{z-z_{0}}-q \frac{\tau^{2}}{z-z_{0}}-r \frac{\tau}{z-z_{0}}=0 .
$$

The solutions to (3.23) are

\footnotetext{
${ }^{10}$ In complex notation Green's theorem for any real-analytic function $F$ defined on $R /\left\{z_{0}\right\}$ reads

$$
\int_{R} \partial \bar{\partial} F \frac{i}{2} d z \wedge d \bar{z}=\frac{i}{4}\left(\oint_{\partial R} \bar{\partial} F d \bar{z}-\oint_{\partial R} \partial F d z\right)
$$
}




$$
\begin{array}{ll}
e^{2 \pi i \tau / p}=z-z_{0} & \text { for } \operatorname{det} Q=0 \text { and } q=r=0, \\
c\left(\frac{\tau-\tau_{0}}{\tau-\bar{\tau}_{0}}\right)^{\frac{\pi}{\sqrt{\operatorname{det} Q}}=z-z_{0}} \quad \text { for } \operatorname{det} Q>0 \text { and } q \neq 0,
\end{array}
$$

where $Q$ is the matrix defined in (2.6),

$$
\tau_{0}=-\frac{r}{2 q}+\frac{i}{|q|} \sqrt{\operatorname{det} Q},
$$

and $c \neq 0$ is a constant. Any $P S L(2, \mathbb{Z})$ transformation of these solutions is again a solution, leading to solutions for all possible values of $p, q$ and $r$.

All these solutions turn out to have a non-trivial monodromy, as we assumed in the previous section. It is always given by

$$
\tau \rightarrow e^{Q} \tau \quad \text { where } \quad e^{Q}=\cos (\sqrt{\operatorname{det} Q}) I+\frac{\sin (\sqrt{\operatorname{det} Q})}{\sqrt{\operatorname{det} Q}} Q .
$$

This identifies $e^{Q}$ as the monodromy matrix and establishes the relation between monodromy and the $p, q, r$ charges of the sources. The two eigenvalues of $e^{Q}$ are $e^{ \pm i \sqrt{\operatorname{det} Q}}$. Note that $\tau_{0}$ is a fixed point of the monodromy matrix $e^{Q}$ when $\operatorname{det} Q>0$. Equation (3.23) also has solutions with $\operatorname{det} Q<0$. We do not consider these solutions here because in dividing out type IIB by $S L(2, \mathbb{Z})$ or any subgroup thereof, points which are fixed points under $e^{Q}$ with $\operatorname{det} Q<0$ do not appear.

The left hand-side of expressions (3.24) and (3.25) can be recognized as expansions of modular functions [27] around fixed points of some modular group of transformations. In sections 5 and 6 we will discuss the full solutions to the scalar field equations (3.21) in terms of such modular functions and incorporate the above solutions as approximations around certain fixed points.

\subsection{The Einstein equations of motion}

Varying the action (2.1) with respect to the metric and substituting equations (3.9) and (3.10) one finds that the $z \bar{z}$ component of the Einstein equations is given by

$$
\partial \bar{\partial} \log |f|^{2}=-\frac{1}{2} \delta\left(z-z_{0}, \bar{z}-\bar{z}_{0}\right) \frac{i}{\tau-\bar{\tau}}\left(p+q|\tau|^{2}+r \frac{\tau+\bar{\tau}}{2}\right),
$$

where $\partial=\frac{\partial}{\partial z}$. All other components of the Einstein equation are identically zero.

Integrating equation (3.28) over a disk $R$ which is bounded by $\gamma_{\delta}$ as defined in section 3.1 and using that $\bar{\partial} f=0$ we obtain

$$
\lim _{\delta \rightarrow 0} \operatorname{Im} \oint_{\gamma_{\delta}}(\log f)^{\prime} d z=-\frac{i}{\tau-\bar{\tau}}\left(p+q|\tau|^{2}+r \frac{\tau+\bar{\tau}}{2}\right)_{z=z_{0}}
$$


Using equations (3.24) and (3.25) we can write

$$
\lim _{\delta \rightarrow 0} \operatorname{Im} \oint_{\gamma_{\delta}}(\log f)^{\prime} d z=-\operatorname{sign}(q) \sqrt{\operatorname{det} Q},
$$

where $e^{Q}$ is the monodromy matrix of $\tau$ measured when going around the contour $\gamma_{\delta}$.

The orders of the zeros/poles of the function $f(z)$ at $z=z_{0}$ determines the deficit angle $\delta$ at the location of the source. Let $\gamma_{\eta}$ be a closed circular contour of radius $\eta$ which encircles the point $z_{0}$. Then we have

$$
\delta=-\frac{1}{i} \lim _{\eta \rightarrow 0} \oint_{\gamma_{\eta}}(\log f)^{\prime} d z .
$$

Combining eqs. (3.30) and (3.31) we thus find the following expression for the deficit angle at the location of the source:

$$
\delta=\operatorname{sign}(q) \sqrt{\operatorname{det} Q} .
$$

Note that there is no deficit angle at the position of a $\operatorname{det} Q=0$-brane.

We will now derive an expression for the mass of the 7-brane solution in terms of a bulk contribution and the deficit angles at the position of the $\operatorname{det} Q>0$ objects. For this purpose we consider the 7 -branes to be wrapped on a $T^{7}$ with radii $R^{1}, \cdots, R^{7}$ so they can be viewed as point-particles moving in a $1+2$-dimensional space-time.

In general, the total energy of a massive particle in $1+2$ dimensions is measured by the deficit angle at infinity via the formula [25]

$$
m=\frac{1}{16 \pi G_{N}^{(3)}} \int d^{2} x \sqrt{|\gamma|} R(\gamma)
$$

where $G_{N}^{(3)}$ is the $(2+1)$-dimensional Newton's constant, related to the 10-dimensional one by

$$
G_{N}^{(3)}=\frac{G_{N}^{(10)}}{(2 \pi)^{7} R^{1} \cdots R^{7}}
$$

and $\gamma$ is the metric of the transverse space.

For static solutions in $2+1$ dimensions one has $G_{0}^{0}=-\frac{1}{2} R(\gamma)$, where $R(\gamma)$ is the Ricci scalar of the metric $\gamma$. We have $G^{0}{ }_{0}=\frac{1}{2} T_{0}^{0}$ and hence the energy is given by

$$
m=\frac{1}{16 \pi G_{N}^{(3)}} \int d^{2} x \sqrt{|\gamma|} R(\gamma)=-\frac{1}{16 \pi G_{N}^{(3)}} \int \frac{i}{2} d z \wedge d \bar{z} \sqrt{|\gamma|} T_{0}^{0} .
$$

We have

$$
T_{0}^{0}=-\frac{1}{\sqrt{|\gamma|}} \frac{1}{(\operatorname{Im} \tau)^{2}} \partial \tau \bar{\partial} \bar{\tau}-\sum_{n} \frac{1}{\sqrt{|\gamma|}} \delta\left(z-z_{n}, \bar{z}-\bar{z}_{n}\right) \frac{1}{\operatorname{Im} \tau}\left(p+q|\tau|^{2}+r \frac{\tau+\bar{\tau}}{2}\right)
$$


where $n$ labels the points $z_{n}$ where the particles are located. Using eqs. (3.29), (3.30) and (3.32) we obtain the following expression for the energy:

$$
m=\frac{1}{16 \pi G_{N}^{(3)}}\left(\int \frac{i}{2} d z \wedge d \bar{z} \frac{\partial \tau \bar{\partial} \bar{\tau}}{(\operatorname{Im} \tau)^{2}}+2 \sum_{n} \delta_{n}\right),
$$

where $\delta_{n}$ is the deficit angle at the location of the $n$th particle at the point $z_{n}$. Branes with $\operatorname{det} Q=0$ do not contribute to the sum in (3.37). Their energy is solely given by the bulk contribution. A further discussion of this can be found in section 5 .

\section{The BPS Equation}

In this section we will derive a BPS equation for 7 -brane solutions relating the energy $m$ to the monodromy of an image object located at the asymptotic region of the transverse space. An image object [25] is an unphysical object which one adds to the solution in order to identify the transverse space with a sphere (one point compactification). By consistency, its charges (including the mass) must "neutralize" the solution. In this case, this means that the monodromy of the image object, which we will denote by $e^{Q_{\infty}}$, is the inverse of the total monodromy measured when going around all other objects. In addition its mass is such that the total deficit angle adds up to $4 \pi$ (the transverse space has become a sphere).

The asymptotic region of the transverse space, the region $|z| \rightarrow \infty$, corresponds to a single point on the Riemann sphere, the point $z=\infty$. This particular point on the Riemann sphere is the location of the image object11. The asymptotic expansion of the metric (3.9) generally takes the form

$$
d s_{\infty}^{2}=-d t^{2}+d \vec{x}_{7}^{2}+\operatorname{cst}|z|^{-8 G_{N}^{(3)} m} d z d \bar{z}
$$

where $\tau_{\infty}=$ cst is the asymptotic value of $\tau$ and where $f \rightarrow z^{-4 G_{3} m}$ near $z=\infty$ with $m \geq 0$ the total mass of the solution. The space is asymptotically conical with deficit angle $\delta=8 \pi G_{N}^{(3)} m$. Not all contributions in (3.37) to the total mass $m$ need to be positive. It is a special property of $(2+1)$-dimensional space-times that one can allow for negative deficit angles (negative point masses). In terms of the function $f$ this statement is no other than saying that $f$ can have both zeros (positive deficit angle) and poles (negative deficit angle). We restrict to solutions for which the total mass $m \geq 0$.

From equation (4.1) it is clear that the point $z=\infty$ must be a zero of the function $f$. The energy (3.37) can be computed as follows

$$
m=\frac{1}{8 \pi G_{N}^{(3)}} \operatorname{Im} \oint_{z=\infty}(\log f)^{\prime} d z
$$

\footnotetext{
${ }^{11}$ Note that the location of the image object is arbitrary. We could have chosen to place it at any other point, $z_{0}$ say, of the Riemann sphere. Generally speaking a point $z_{0}$ on the Riemann sphere is mapped to an asymptotic region of the transverse space when the physical distance from $z_{0}$ to any other point diverges as $|z|^{1-4 G_{N}^{(3)} m}$ while $m<1 / 4 G_{N}^{(3)}[25]$.
} 
where the contour integral encircles the point $z=\infty$ (in a counter-clockwise direction). We must have, in order that $\epsilon$ transforms correctly when going around infinity, that

$$
m=\frac{1}{8 \pi G_{N}^{(3)}} \operatorname{Im} \oint_{z=\infty}(\log f)^{\prime} d z=-\operatorname{sign}\left(q_{\infty}\right) \frac{\sqrt{\operatorname{det} Q_{\infty}}}{8 \pi G_{N}^{(3)}}=-\frac{\delta_{\infty}}{8 \pi G_{N}^{(3)}},
$$

where $Q_{\infty}$ is the charge matrix of the monodromy of $\tau$ when going around $z=\infty$. Equation (4.3) may be referred to as the BPS identity for 7-brane solutions. Note that, in order that $m>0$ we must have $\delta_{\infty}<0$.

It is instructive to compare the BPS identity (4.3) with the mass formula (3.37). The identification of the two formulae implies

$$
\int \Omega=-2 \sum_{n^{\prime}} \delta_{n^{\prime}}
$$

where the sum is extended to all "holes" in the transverse space, including the asymptotic region and the 1.h.s. is the integral of the pull-back of the Kähler 2-form defined in (3.7). This, in turn, implies that the deficit angles at the locations of the particles/7-branes in transverse space, including the image particle, can also be computed via line integrals of the pull-back of the Kähler connection around the locations of the particles/7-branes in transverse space

$$
\delta=\frac{1}{2} \oint \mathcal{Q}
$$

This expression can be taken as the definition of $U(1)$ charge (see [30]). If we apply this formula to compute the $U(1)$ charge at infinity (4.3) becomes a relation between the mass and the $U(1)$ charge with the characteristic form of a (saturated) BPS bound. It is now not too difficult to see that the proof of [30] that all the solutions of the system under consideration which have the asymptotic behavior that allows to define mass and $U(1)$ charge are automatically supersymmetric and both are related by the saturated BPS bound which becomes an identity. In other words, there are no "black" 7-brane solutions with a horizon.

Finally, observe that (4.5) can be written in the form

$$
\delta=\frac{1}{2} \operatorname{Im} \oint(\log \operatorname{Im} \tau)^{\prime},
$$

and, comparing with (3.31) we see that the fact that $\delta$ can be computed using either $\operatorname{Im} \tau$ or $f$ is a consequence of both functions having related monodromies, which is something we required in order to have well-defined Killing spinors and supersymmetry.

\section{Constructing Solutions}

In this section we will discuss how to construct globally well-defined solutions. The 7brane configuration (3.9), (3.10) contains two undetermined holomorphic functions: $\tau(z)$ 
and $f(z)$. Both functions are defined on the Riemann sphere, $\hat{\mathbb{C}}$.

An important role in constructing a globally well-defined solution is the choice of the monodromy group which we will discuss first. Consider an arbitrary point $b \in \hat{\mathbb{C}}$ and form all possible closed loops with $b$ as their common base point. The set of all monodromies measured when going around each of these loops forms a group, called the monodromy group. The function $\tau$ transforms under $\operatorname{PSL}(2, \mathbb{Z})$ and the function $f$ transforms under $S L(2, \mathbb{Z})$. By monodromy group we will always mean the monodromy group of $\tau$.

Consider a single D7-brane. This corresponds to $p=1$ in equation (3.24). The monodromy of $\tau$ measured when going around a single D7-brane is $\tau \rightarrow T \tau \equiv \tau+1$, where $T$ is defined in (3.4). Further, from equation (3.17) it follows that $f \rightarrow f$. The element $T \in P S L(2, \mathbb{Z})$ is of infinite order. However, solutions containing only one object with this monodromy will have infinite mass per volume element [1]. This is related to the fact that after modding out the complex plane with $T$ the resulting fundamental domain has infinite area (measured with respect to $\frac{i}{2} \frac{d \tau \wedge d \bar{\tau}}{(\operatorname{Im} \tau)^{2}}$ ), and this leads to an infinite mass per volume element, see (5.2). Thus, to obtain solutions of finite mass, we are forced to include objects with other monodromies. Here we employ the S-duality of the theory.

We will focus on solutions whose monodromy group is $\operatorname{PSL}(2, \mathbb{Z})$ which is generated by $T$ and $S$. To show that one can also work with subgroups we indicate in subsection 6.4 the construction of such solutions for the specific case of the group $\Gamma_{0}(2)$ whose generators are $T$ and $S T^{2} S$. Having chosen a monodromy group we can specify the functions $\tau(z)$ and $f(z)$.

\subsection{The function $\tau(z)$}

Since $\tau$ and $\Lambda \tau$ are identified we need a function, $j(\tau)$, which is monodromy neutral, i.e. is an automorphic function of the monodromy group

$$
j(\Lambda \tau)=j(\tau)
$$

where $\Lambda$ is any element of that group. The local expansions of the function $j$ around the fixed points of $\Lambda$ are as given in (3.24) and (3.25).

A region of the complex upper half plain containing values of $\tau$ which are inequivalent under the monodromy group but which are related to all the points in the upper half plane is a fundamental domain of the monodromy group. Note that the fundamental domain is in general an orbifold. Points which are fixed points under some elements of the group are called orbifold points. In our examples we always deal with a total of three orbifold points. In figure 1 and table 2 we have summarized some properties of the monodromy groups $P S L(2, \mathbb{Z})$ and $\Gamma_{0}(2)$ and the standard choices for their fundamental domains (see, e.g. [31].)

We require that $j(\tau)$ maps the fundamental domain onto the Riemann sphere $\hat{\mathbb{C}}$ in a one-to-one fashion, so that the inverse function $j^{-1}$ exists. The function $\tau(z)$ is then given by $\tau(z)=j^{-1}(z)$. Often we will include a further map from the Riemann sphere to $N$ copies of itself which is given by the $N$ to 1 automorphism $z \rightarrow P(z) / Q(z)$ for polynomials 
$P(z)$ and $Q(z)$. For $N=1$ these polynomials are fixed by the requirement that the three orbifold points of the fundamental domain are mapped to three given points in the $z$-plane, which can always be achieved by an $S L(2, \mathbb{C})$ transformation. For instance, the modular $j$ function maps the points $\{i \infty, \rho, i\}$ to $\{\infty, 0,1\}$ with $\rho=-\frac{1}{2}+\frac{i}{2} \sqrt{3}$. Similarly the function $j_{\Gamma_{0}(2)}$ maps the points $\{i \infty, \sigma, 0\}$ to $\{\infty, 0,1\}$ with $\sigma=-\frac{1}{2}+\frac{i}{2}$. For $N>1$ the polynomials $P(z)$ and $Q(z)$ are fixed by the further requirement of how many branes are placed at the three points $z_{i \infty}, z_{\rho}$ and $z_{i}$ where the subscript indicates the value of $\tau$ at that point. For $N=1$ there is one brane at each point. In the next section we will give explicit realizations of $P(z)$ and $Q(z)$. Note that, for general $N$, the mass formula (3.37) becomes

$$
m=\frac{1}{16 \pi G_{N}^{(3)}}\left(N \times \text { area fundamental domain }+2 \sum_{j} \delta_{j}\right),
$$

where the area is measured with the area element

$$
\frac{i}{2} \frac{d \tau \wedge d \bar{\tau}}{(\operatorname{Im} \tau)^{2}} .
$$

Summarizing, we have the sequence of maps

$$
z \quad \stackrel{N \rightarrow 1}{\longrightarrow} \quad \frac{P(z)}{Q(z)} \quad \stackrel{j^{-1}}{\longrightarrow} \quad \tau(z)=j^{-1}\left(\frac{P(z)}{Q(z)}\right) .
$$

The inverse mapping $j^{-1}$ which maps from the Riemann sphere $\hat{\mathbb{C}}$ onto the fundamental domain has branch cuts connecting the points $z_{i \infty}$ to $z_{\rho}$ and $z_{\rho}$ to $z_{i}$. Likewise the inverse function $j_{\Gamma_{0}(2)}^{-1}$ has branch cuts connecting the points $z_{i \infty}$ to $z_{\sigma}$ and $z_{\sigma}$ to $z_{0}$.
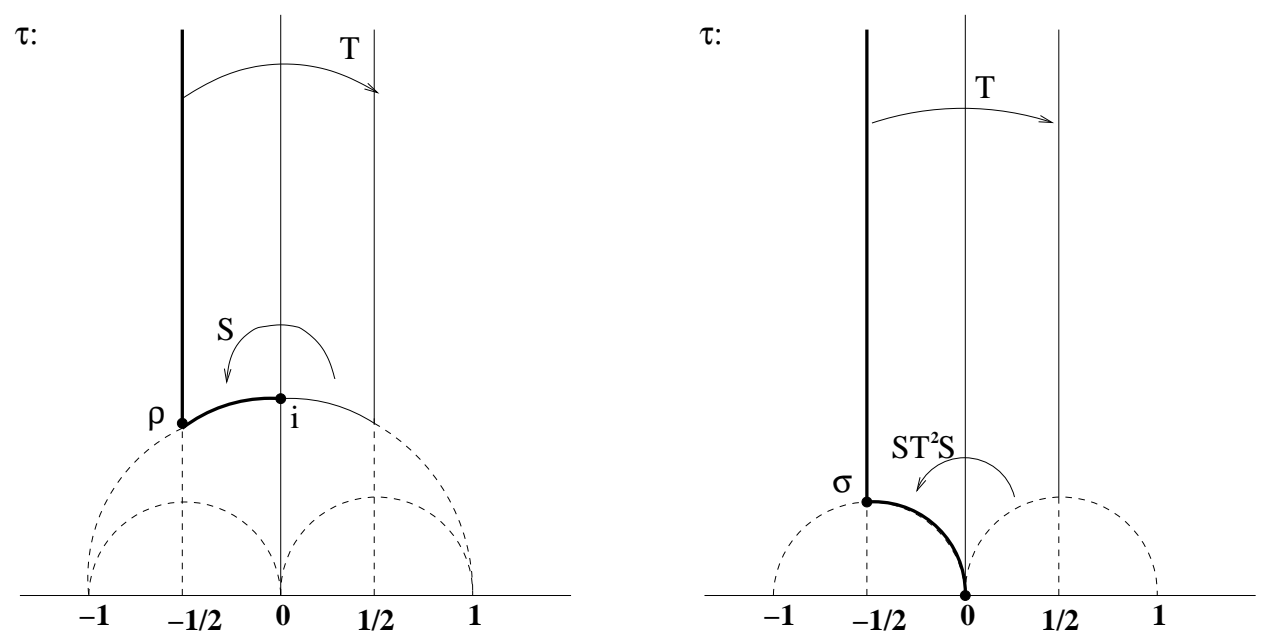

Figure 1: The fundamental domains of the groups $P S L(2, \mathbb{Z})$ and $\Gamma_{0}(2)$, respectively. The points $\rho$ and $\sigma$ denote the points $-\frac{1}{2}+\frac{i}{2} \sqrt{3}$ and $-\frac{1}{2}+\frac{i}{2}$, respectively. 


\begin{tabular}{||c||c|c|c||c|c||}
\hline & generators & orbifold pts. & area & $j(\tau)$ & $F(\tau)$ \\
\hline \hline $\operatorname{PS} L(2, \mathbb{Z})$ & $T, S$ & $i \infty, \rho, i$ & $\pi / 3$ & Klein's modular $j$ function $[26]$ & $\eta^{2}(\tau)$ \\
\hline$\Gamma_{0}(2)$ & $T, S T^{2} S$ & $i \infty, 0, \sigma$ & $\pi$ & $j_{\Gamma_{0}(2)} \equiv \frac{1}{(1+i)^{12}}\left(\frac{\eta(\tau)}{\eta(2 \tau)}\right)^{24}$ & $\eta(\tau) \eta(2 \tau)$ \\
\hline
\end{tabular}

Table 2: Properties of the groups $P S L(2, \mathbb{Z})$ and $\Gamma_{0}(2)$ and corresponding realizations of the functions $j(\tau)$ and $F(\tau)$.

\subsection{The function $f(z)$}

The function $f(z)$ can be written in the form

$$
f(z)=F(\tau) h(z),
$$

where $F(\tau)$ is a modular function which transforms under $P S L(2, \mathbb{Z})$ as

$$
F\left(\frac{a \tau+b}{c \tau+d}\right)=e^{i \beta(a, b, c, d, \tau)}(c \tau+d) F(\tau),
$$

and $h(z)$ is a function of $z$ which we choose such that when going around a 7-brane it transforms as

$$
h(z) \rightarrow e^{i k \pi} e^{-i \beta(a, b, c, d, \tau(z))} h(z)
$$

where $k=0,1$. For $k=0$ we have a plus sign and this must be used for transformations under the identity of $S L(2, \mathbb{Z})$. For $k=1$ we have a minus sign which must be used for transformation under the element $-\mathbb{1}$ of $S L(2, \mathbb{Z})$. This additional sign is necessary because $f$ transforms under $S L(2, \mathbb{Z})$. It will play an important role later in the construction of solutions. We see that $f$ transforms under $S L(2, \mathbb{Z})$ as $\pm \mathbb{1} \times P S L(2, \mathbb{Z})$ and that the $\pm \mathbb{1}$ part is independent of $\tau$.

When going around a D7-brane the function $f$ does not change, so that $f$ has no zero/pole at the point $z=z_{i \infty}$. This follows from equation (3.13). We do however expect to measure a positive contribution to the total energy when going to infinity, $|z| \rightarrow \infty$, due to the presence of a D7-brane. Therefore, $f$ must lead to a non-trivial deficit angle at infinity. Indeed, we know that $f(z) \rightarrow z^{-4 G_{N}^{(3)} m}$ for $|z| \rightarrow \infty$. This asymptotic behaviour must follow from the function $h(z)$ since $\tau_{\infty}=$ cst and therefore $F(\tau)$ does not contribute. Around $z=z_{i \infty}$ this leads to a factor $\left(z-z_{i \infty}\right)^{-\alpha}$ with $\alpha>0$ in $h(z)$, and therefore to a pole in $h(z)$ for $z=z_{i \infty}$. Since we just argued that $f(z)$ cannot have such a pole, it must be cancelled by a zero of $F$ at $\tau=i \infty$. We have thus established that $F(\tau)$ must be a cusp form. A lot is known about such cusp forms in the mathematical literature. The explicit realizations of these cusp forms in terms of the Dedekind eta function $\eta(\tau)$ for the groups 
$P S L(2, \mathbb{Z})$ and $\Gamma_{0}(2)$ is given in table 2. Using the monodromies of this cusp form and the required monodromies of $f(z)$ it is not difficult to derive an explicit realization of the function $h(z)$.

The choice of the function $h(z)$ is case dependent. We will give explicit expressions in section 6. Here we only give the transformation of the Dedekind $\eta$-function under the different $P S L(2, \mathbb{Z})$-transformations:

$$
\begin{aligned}
T: \eta^{2}(\tau+1) & =e^{\pi i / 6} \eta^{2}(\tau), \\
S: \quad \eta^{2}\left(-\frac{1}{\tau}\right) & =e^{-\pi i / 2} \tau \eta^{2}(\tau), \\
T^{-1} S: \quad \eta^{2}\left(-\frac{\tau+1}{\tau}\right) & =e^{-2 \pi i / 3} \tau \eta^{2}(\tau) .
\end{aligned}
$$

For the convenience of the reader we present in table 3 the monodromies of $\tau$ and $f$ measured when going around the points $z_{i \infty}, z_{\rho}, z_{i}$ in a counter clockwise direction and the deficit angles. Instead of comparing monodromies it is sometimes convenient to compare deficit angles.

This completes the construction of a globally well-defined 7-brane solution. We will present several explicit examples in the next section.

\section{Examples of Solutions}

In this section we give several examples of globally supersymmetric solutions using the ingredients discussed so far. In particular, we will analyze the global properties of the Killing spinor. This analysis will determine the precise form of the function $h(z)$.

We will concern ourselves mostly with the monodromy group $S L(2, \mathbb{Z})$. Only in the last subsection 6.4 an example with the monodromy group $\Gamma_{0}(2)$ will be discussed.

\subsection{Solutions containing a single D7-brane}

We first present the simplest possible solution containing a single D7-brane using the results of the previous sections. In the next subsection we will argue that the solutions of this subsection can be viewed as special limits of more general solutions. The latter solutions which we refer to as the basic building blocks can be used to generate all possible 7-brane solutions containing an arbitrary number of D7-branes.

One way to derive an explicit form for $h(z)$ is to compare monodromies. We first consider a D7-brane which is located at the point $z_{i \infty}$. The monodromy of $f=\eta^{2}$ around the point $z_{i \infty}$ is $f \rightarrow e^{\pi i / 6} f$, according to eq. (5.8). This does not coincide with the transformation required by $S L(2, \mathbb{Z})$, which is $f \rightarrow f$. For this reason one should include 


\begin{tabular}{||c||c|c|c|c||}
\hline location & $S L(2, \mathbb{Z})$ & $(p, q, r)$ & monodromy $f$ & deficit angle $\delta$ \\
\hline \hline$z_{i \infty}$ & $T$ & $(1,0,0)$ & $f \rightarrow f$ & 0 \\
\hline$z_{i}$ & $S$ & $(-\pi / 2,-\pi / 2,0)$ & $f \rightarrow \tau f$ & $-\pi / 2$ \\
\hline$z_{i}$ & $-S$ & $(\pi / 2, \pi / 2,0)$ & $f \rightarrow e^{i \pi} \tau f$ & $\pi / 2$ \\
\hline$z_{\rho}$ & $T^{-1} S$ & $\left(-\frac{4 \pi}{3 \sqrt{3}},-\frac{4 \pi}{3 \sqrt{3}},-\frac{4 \pi}{3 \sqrt{3}}\right)$ & $f \rightarrow \tau f$ & $-2 \pi / 3$ \\
\hline$z_{\rho}$ & $-T^{-1} S$ & $\left(\frac{2 \pi}{3 \sqrt{3}}, \frac{2 \pi}{3 \sqrt{3}}, \frac{2 \pi}{3 \sqrt{3}}\right)$ & $f \rightarrow e^{i \pi} \tau f$ & $\pi / 3$ \\
\hline
\end{tabular}

Table 3: The monodromy of $\tau$ and $f$, the $p, q, r$ values and the deficit angles for $\tau=$ $i \infty, \rho, i$. The deficit angle $\delta$ is computed using $\delta=\operatorname{sign}(q) \sqrt{\operatorname{det} Q}$. In the monodromy transformation for $f$ we take $1=e^{i 0}$ and $-1=e^{i \pi}$ in agreement with the discussion below equation (5.7) about the number $k=0,1$.

a factor $\left(z-z_{i \infty}\right)^{-1 / 12}$ in $f$, i.e. $h(z) \sim\left(z-z_{i \infty}\right)^{-1 / 12}$. With this choice of $f$ the behaviour of the Killing spinor around the point $z_{i \infty}$ coincides with the $S L(2, \mathbb{Z})$ requirement.

Branes located at the points $z_{i}$ and $z_{\rho}$ are named after their monodromy under $S L(2, \mathbb{Z})$. For example, if we consider a point $z_{i}$ with $S$ monodromy then we call this an $S$-brane. For such a brane we must have $f \rightarrow \tau f$. From equation (5.9) it follows that around $z_{i}$ the function $h$ must transform as $h \rightarrow e^{i \pi / 2} h$, so we include an additional factor $\left(z-z_{i}\right)^{1 / 4}$. For a $(-S)$-brane one must include a factor of $\left(z-z_{i}\right)^{-1 / 4}$. If we consider a brane at $z_{\rho}$ with $T^{-1} S$ monodromy then $f \rightarrow \tau f$. In order to compensate the factor which appears in (5.10) we must include a factor $\left(z-z_{\rho}\right)^{1 / 3}$. Likewise for a brane with $-T^{-1} S$ monodromy the factor which appears in $h$ is $\left(z-z_{\rho}\right)^{-1 / 6}$.

If we take $z_{\rho}=\infty$, which means that the asymptotic value of $\tau$ is $\rho$, the simplest solution is given by

$$
j=\frac{\left(z_{i}-z_{i \infty}\right)}{\left(z-z_{i \infty}\right)}, \quad f=\eta^{2}\left(z-z_{i \infty}\right)^{-1 / 12}\left(z-z_{i}\right)^{-1 / 4}
$$

This solution is asymptotically conical with a deficit angle of $2 \pi / 3$. If on the other hand $z_{i}=\infty$, so that the asymptotic value of $\tau$ is equal to $i$ then we find 


$$
j=\frac{\left(z-z_{\rho}\right)}{\left(z-z_{i \infty}\right)}, \quad f=\eta^{2}\left(z-z_{i \infty}\right)^{-1 / 12}\left(z-z_{\rho}\right)^{-1 / 6} .
$$

This is asymptotically a cone with deficit angle $\pi / 2$. The properties of solutions of this type will be discussed further at the end of the next subsection.

\subsection{The basic building blocks}

In order to consider more general solutions for $N>1$ it would be convenient to have a means of obtaining them directly starting from the $N=1$ solutions. The above method of constructing solutions via monodromy requirements becomes cumbersome for large $N$. In this subsection we will construct two basic solutions, the $N=1 A$ and $N=1 B$ solutions also called the basic building blocks, out of which any other solution can be generated in a manner to be described shortly. In particular the solutions presented in the previous subsection appear as special limits of these $N=1 A$ and $N=1 B$ solutions.

The problem in using (6.1) and (6.2) as the starting point for higher $N$ solutions is that they have a nontrivial asymptotic geometry. We can however add a point mass to a solution whose mass is equal and opposite to the total mass measured at infinity. This is because point masses in $2+1$ dimensions only form deficit angles, but otherwise do not deform the solution. The resulting space is then by construction asymptotically flat and has total mass zero. In terms of monodromies the idea is thus to start with a solution in which all points around which there is a nontrivial monodromy are at finite values of $z$. So there is no monodromy for $\tau$ and $f$ around the point $z=\infty$.

For $N=1$ two such configurations are possible 12 . The first consists of three branes with monodromies $T, S$ and $-T^{-1} S$ and the following choice for the functions $j$ and $f$ :

$$
1 A: \quad j=\frac{\left(z-z_{\rho}\right)\left(z_{i}-z_{i \infty}\right)}{\left(z-z_{i \infty}\right)\left(z_{i}-z_{\rho}\right)}, \quad f=\eta^{2}\left(z-z_{i \infty}\right)^{-1 / 12}\left(z-z_{i}\right)^{1 / 4}\left(z-z_{\rho}\right)^{-1 / 6} .
$$

Due to the zeroes and poles of $f$, there are deficit angles (and hence masses) at the points $z_{i}$ and $z_{\rho}$ which are given by $-\pi / 2$ and $\pi / 3$, respectively. Note that the brane with $S$ monodromy has a negative mass and deficit angle, and that the total mass adds up to zero:

$$
m \sim \frac{\pi}{3}+\frac{2 \pi}{3}-\pi=0 .
$$

We will refer to this solution as the $N=1 A$ solution. In the limit in which the negative mass brane is sent to the asymptotic region $13 z_{i} \rightarrow \infty$ the solution becomes equation (6.2).

\footnotetext{
${ }^{12}$ We do not consider the possibility of having branes with $-T$ monodromy because $-T$ is not continuously related to the identity in $S L(2, \mathbb{Z})$.

${ }^{13}$ To take this limit one must rescale $f$ in $(6.3)$ with a factor $z_{i}^{-1 / 4}$ in order that this $f$ goes to the $f$ of (6.2).
} 
Whenever one constructs solutions in this way such a limiting procedure must always be performed. The procedure should be considered as a solution generating technique. It does not represent some kind of physical process.

The method used here is somewhat similar to the method of images used in section 4 . The difference is that here we are considering solutions that are asymptotically $\mathbb{R}^{2}$ whereas the image object gives rise to $S^{2}$. Using the latter method the 1 A solution would have contained the factor $\left(z-z_{i}\right)^{-2+1 / 4}$ instead of $\left(z-z_{i}\right)^{1 / 4}$ leading to a total deficit angle $4 \pi$. In this case the point $z_{i}$ satisfies the criterion given in footnote 11 of section 4 and should be considered as the asymptotic region. In the present case the point $z_{i}$ does not satisfy this criterion and it should be considered as the location of an actual object with negative deficit angle equal to $\delta_{\infty}$ (see equation (4.3)). In order to do away with it one has to take a singular limit as described in footnote 13.

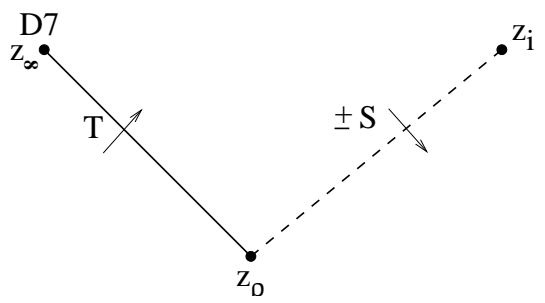

Figure 2: The $N=1 A$ and $N=1 B$ supersymmetric 7-brane solutions, with the D'-brane at $z_{i \infty}$ and $\operatorname{det} Q>0$-branes at the points $z_{\rho}$ and $z_{i}$. The filled (dashed) lines are $T(S)$

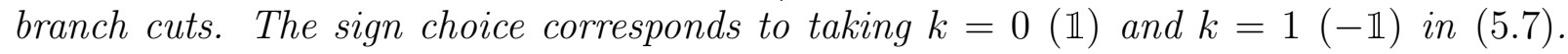
The $N=1 A$ and $N=1 B$ solutions have upper and lower signs, respectively.

The second possibility consists of branes with monodromies $T,-S$ and $T^{-1} S$. Note that these only differ in a number of signs from the previous one. Since the scalars are only sensitive to the $P S L(2, \mathbb{Z})$ part the function $j$ remains unchanged. The Killing spinor is sensitive to the signs and therefore the function $f$ is different in this case. It is given by

$$
1 B: \quad f=\eta^{2}\left(z-z_{i \infty}\right)^{-1 / 12}\left(z-z_{i}\right)^{-1 / 4}\left(z-z_{\rho}\right)^{1 / 3} .
$$

The poles and zeroes of $f$ now give rise to deficit angles of $\pi / 2$ and $-2 \pi / 3$ at the points $z_{i}$ and $z_{\rho}$, respectively. Note that in this case the object at $z_{\rho}$ has a negative mass and again the total mass is vanishing:

$$
m \sim \frac{\pi}{3}-\frac{4 \pi}{3}+\pi=0 .
$$

This solution will be referred to as the $N=1 B$ solution. The limit $14 z_{\rho} \rightarrow \infty$ gives rise to equation (6.1). The $1 \mathrm{~A}$ and $1 \mathrm{~B}$ solutions are pictorially represented as in figure 2 .

One should think of figure 2 as being the Riemann sphere parameterized by $z$. When crossing a branch cut we go to a different sheet on the Riemann sphere. Lines of $T$

\footnotetext{
${ }^{14}$ In this case one must first multiply $f$ by a factor $z_{\rho}{ }^{-1 / 3}$ before taking the limit.
} 
monodromy connect one point $z_{i \infty}$ to one point $z_{\rho}$ and lines of $S$ monodromy connect one point $z_{\rho}$ to one point $z_{i}$. Just from the order of the branch cuts one can uniquely construct the function $j(\tau)$ from the figure. In order to also construct the function $f$ we have indicated the appropriate signs which play a role in determining the function $h$ as explained below equation (5.7). In other words the figure is a unique pictorial representation of the pair $(\tau, f)$. A particularly convenient property of such a representation is that it also captures the global positioning of the branch cuts and thus the monodromy measured when going along large loops. This should be contrasted with equation (3.27) where the monodromy of $\tau$ is computed for loops which go around a brane at an infinitesimal distance.

Solutions with higher $N$ can now be constructed by combining any given number of the $1 \mathrm{~A}$ solutions with any given number of the $1 \mathrm{~B}$ solutions. For example, for $N=2$ one can combine two $N=1$ solutions of the same or different types, yielding three different possibilities. The combination of e.g. two $1 A$ solutions leads td 15

$$
j=\lambda \prod_{n=1,2} \frac{\left(z-z_{\rho}^{(n)}\right)}{\left(z-z_{i \infty}^{(n)}\right)}, \quad f=\eta^{2} \Pi_{n=1,2}\left(z-z_{i \infty}^{(n)}\right)^{-1 / 12}\left(z-z_{i}^{(n)}\right)^{1 / 4}\left(z-z_{\rho}^{(n)}\right)^{-1 / 6}
$$

where $\lambda$ is some complex constant. The two points $z_{i}^{(n)}$ are given by the roots of the equation $j=1$ and depend on the value of $\lambda$. The other two possibilities with $N=2$ require the obvious changes in the powers of $\left(z-z_{i}\right)$ and $\left(z-z_{\rho}\right)$ in the function $f$. Higher$N$ solutions are built along the same lines. Equation (6.7) shows the advantage of working in a space of total deficit angle zero as opposed to one of total deficit angle of $4 \pi$ because only in the former case can we write the function $h$ which appears in $f$ as a product of $h(1 A)$ and $h(1 B)$.

Several remarks are in order. In the 7-brane solutions of $[1,3]$ the largest number of D7-branes which a solution can contain is 24 because for this number the transverse space has become a sphere. It is now possible to construct solutions with more than 24 D7-branes if and only if one allows for the presence of $\operatorname{det} Q>0$-branes with negative mass. Further, in order to have the value of $\tau$ at infinity arbitrary so that the asymptotic region can be taken as an approximation of perturbative string theory we need to take combinations of the $1 \mathrm{~A}$ and $1 \mathrm{~B}$ solutions such that there is one point whose $\tau$ monodromy is the identity (in $P S L(2, \mathbb{Z})$ ). This can for example be realized by taking two points with $S$ monodromy coincident and subsequently sending that point to infinity. The third remark is that because $T$ monodromies are of infinite order one cannot eliminate the D7-branes. The reason that the $\operatorname{det} Q>0$-branes can be eliminated is because their monodromies are of finite order.

Note that both $N=1$ solutions are characterized by three complex constants, while the $N=2$ solution has five. A general solution containing $N$ D7-branes has $2 N+1$ complex parameters. Equation (3.12) tells us that the metric is fixed up to global $S L(2, \mathbb{C})$ transformations of the complex coordinate $z$. By placing the branes all at finite values of

\footnotetext{
${ }^{15}$ The combination of one $1 A$ and one $1 B$ solution is given in (6.9).
} 
$z$ we have fixed the $z \rightarrow 1 /(z-c)$ element of $S L(2, \mathbb{C})$. By using the freedom to shift and scale $z$ we can eliminate two complex constants leaving $2 N-1$ free. The number of complex parameters $2 N-1$ is further reduced by one if we send the point with negative deficit angle $\delta_{\infty}$ off to infinity. This fixes the $S L(2, \mathbb{C})$ coordinate freedom. From the Killing spinor equation (3.11) we see that we have the additional freedom to scale the absolute value of the function $f$. This would leave the metric invariant if we could compensate by a scaling of $z$, which cannot be done since we have already used up the $S L(2, \mathbb{C})$ coordinate freedom. We leave, then, this modulus free, and we end up with $2(2 N-2)+1$ free real parameters. This number is made out of $2 N-2$ complex parameters and one real parameter. The complex numbers describe the relative positions of the branes and will at the end of this subsection be related to the number of vectors which can exist on these 7-brane configurations.

The background fields of type IIB which may have nontrivial zero modes on the 7-brane

backgrounds are $g_{\mu \nu}, A_{\mu \nu \rho \sigma}^{+}$(self-dual 4-form) and $\tau$. The 2-forms do not appear since in order to construct the solutions we had to divide out type IIB supergravity by $S L(2, \mathbb{Z})$. The fact that $\tau$ is a very special function of $z$, which it must be in order that it takes values in the fundamental domain of $\operatorname{PSL}(2, \mathbb{Z})$, suggests to interpret the excitations of the background as the modes of a supergravity theory in 8 dimensions. It was mentioned in footnote 6, section 3.1, that this theory has $N=1$ supersymmetry and consists of one supergravity and one vector multiplet. The above counting argument seems to suggest that this 8-dimensional supergravity must be coupled to an additional number of $2 N-2$ vector multiplets coming from the number of free complex numbers describing the relative positions of the branes. Notice that for $N \geq 3$ this number is larger than the number of D7-branes, $N$, and hence, there are more vectors than D7-branes. This seems to suggest that one has to attribute some of the vectors to the worldvolume theories of the $\operatorname{det} Q>0$ branes.

\subsection{F-theory solutions}

The well-known 7-brane configurations of F-theory have the property that the monodromy of $\tau$ close to the points $z_{i}, z_{\rho}$ is the identity in $P S L(2, \mathbb{Z})$ and $T$ around $z_{i \infty}$. Further it is required that the function $f$ has no zeros, which can be interpreted as saying that all mass comes solely from the D7-branes. This condition is satisfied if and only if $\tau$ and $f$ are of the following form

$$
j(\tau)=\frac{P^{3}(z)}{P^{3}(z)+Q^{2}(z)}, \quad f=\eta^{2}\left(P^{3}+Q^{2}\right)^{-1 / 12},
$$

where $P^{3}+Q^{2}$ is a polynomial of order $N$ whose zeros are the locations of the D7-branes. Solutions of this type exist whenever $N$ can be divided by either 2 or 3 . Thus they exist for $N=2,3,4,6,8,9,10,12,24$. In F-theory one cannot go beyond $N=24$ because for this value of $N$ the transverse space has become a sphere. Each time the deficit angle at infinity is given by $2 \pi N / 12$. Out of this set of solutions of $\tau$ those which have $N=6,12,24$ are such that the asymptotic value of $\tau$ can take any value. For the other solutions the 
asymptotic value is necessarily either $i$ or $\rho$. Therefore, these are necessarily nonperturbative in nature. Functions $\tau$ which solve equation (6.8) are the modular parameter of an elliptically fibered torus whose base manifold is the transverse space of the D7-branes. The geometric interpretation of $f$ is that $f d z d \tau$ becomes the holomorphic $(2,0)$-form of the CY two-fold.

F-theory solutions form a subset of our general solutions. They are obtained by having coincident branes of the same type 16 as follows. Take combinations of the $1 \mathrm{~A}$ and $1 \mathrm{~B}$ solutions in which there is only one point $z_{i}$ or $z_{\rho}$ which has a negative deficit angle. Around this special point the monodromy of $\tau$ can be anything. Further, there should be no points $z_{i}$ and $z_{\rho}$ around which either $\tau$ or $f$ has a nontrivial monodromy.

\section{The $N=2,3$ solution with only D7-branes}

Branes at the points $z_{i}$ either have deficit angle $-\pi / 2$ or $+\pi / 2$. Combining two branes one of monodromy $S$ and one of monodromy $S^{-1}=-S$ gives rise to a cancellation of their masses. If we combine the $N=1 A$ and $N=1 B$ solutions without making any assumption about the positions of the branes we have for the function $f$

$$
f=\eta^{2}\left(z-z_{i \infty}^{(1)}\right)^{-1 / 12}\left(z-z_{i \infty}^{(2)}\right)^{-1 / 12}\left(z-z_{i}^{(1)}\right)^{1 / 4}\left(z-z_{i}^{(2)}\right)^{-1 / 4}\left(z-z_{\rho}^{(1)}\right)^{-1 / 6}\left(z-z_{\rho}^{(2)}\right)^{1 / 3} .
$$

The cancellation mechanism just discussed can now be applied by taking the points $z_{i}^{(1)}$ and $z_{i}^{(2)}$ coincident. Next we also take the points $z_{\rho}^{(1)}$ and $z_{\rho}^{(2)}$ coinciding so that we end up with only one point $z_{\rho}$ around which $\tau$ has a nontrivial monodromy. Finally, one must multiply the resulting function $f$ by $z_{\rho}^{-1 / 6}$ and send $z_{\rho} \rightarrow \infty$. This leaves us with the required form for $f$

$$
f=\eta^{2}\left(z-z_{i \infty}^{(1)}\right)^{-1 / 12}\left(z-z_{i \infty}^{(2)}\right)^{-1 / 12} .
$$

Applying these choices to the function $j(\tau)$ for a general $N=2$ solution which is given in (6.9) leads to

$$
j(\tau)=\frac{C}{C+\left(z-z_{i}\right)^{2}},
$$

where $C \neq 0$ is a constant. It is clear that the asymptotic value for $\tau$ is equal to $\rho$. This solution is therefore strictly nonperturbative.

In a similar fashion one can construct the $N=3$ solution. Now one can combine two branes with monodromy $-T^{-1} S$ and one brane of monodromy $T^{-1} S$ at $z_{\rho}$ leading to vanishing mass and trivial monodromy. This is based on the deficit angles $+\pi / 3$ or $-2 \pi / 3$ and the identity $\left(-T^{-1} S\right) \cdot\left(-T^{-1} S\right) \cdot\left(T^{-1} S\right)=\mathbb{1}$. Hence in this case we need to take two $N=1 A$ and one $N=1 B$ solutions. Doing so we have three points $z_{i}$ with monodromies

\footnotetext{
${ }^{16}$ It is not possible to have branes of different type coinciding, since these require different values of $\tau$ at the same point.
} 
$S, S$ and $-S$ that we need to deal with. Taking one brane with $S$ and one brane with $-S$ monodromy coincident we are left with one point $z_{i}$ which has $S$ monodromy and hence a negative deficit angle. Sending this point to infinity we end up with the form of $f$ and $\tau$ as in (6.8). This time the asymptotic value for $\tau$ is equal to $i$.

\section{The $N=6$ solution with only D7-branes}

The case $N=6$ is the first instance in which solutions with only D7-branes and an arbitrary value for $\tau$ at infinity are possible. It can be constructed as follows. From four $N=1 A$ solutions and two $N=1 B$ solutions one can make the following combinations of points:

$$
N=6:\left\{\begin{array}{cc}
\left(-T^{-1} S\right) \cdot\left(-T^{-1} S\right) \cdot\left(T^{-1} S\right)=\mathbb{1}, & \text { at two points } z_{\rho} \\
(-S) \cdot S=\mathbb{1}, & \text { at two points } z_{i} \\
S \cdot S=-\mathbb{1}, & \text { at one point } z_{i}
\end{array}\right.
$$

The function $f$ corresponding to $(6.12)$ is given by

$$
f=\eta^{2}\left(P^{3}+Q^{2}\right)^{-1 / 12}\left(z-z_{i}\right)^{1 / 2} .
$$

Note that four of the five points have total $S L(2, \mathbb{Z})$-monodromy $\mathbb{1}$ : the mass and charge cancel here. The remaining point $z_{i}$ has $-\mathbb{1}$ and there is a negative mass at this point. See figure 3 for an illustration. To obtain a solution with only D7-branes we first multiply $f$ in (6.13) by a factor $z_{i}{ }^{-1 / 2}$ and then send the point $z_{i}$ in $f$ to infinity. It should be mentioned that the value of $\tau$ in the limit $z_{i} \rightarrow \infty$ can now be different from $\tau=i$. The value of $\tau$ after taking the limit depends on the values of the constants appearing in the polynomials $P$ and $Q$.

There is a similar solution composed of three $N=1 A$ and three $N=1 B$ solutions in which there are three $z_{i}$ and one $z_{\rho}$ orbifold point with vanishing mass and trivial monodromy $+\mathbb{1}$. The other point $z_{\rho}$ again has a monodromy $-\mathbb{1}$ and a negative mass. In fact this solution is equivalent to the $N=6$ solution above: they differ in the assignation of signs to the separate $N=1 A$ and $N=1 B$ solutions but these all cancel in this particular configuration. In what follows we will use the first parametrization of this solution.

A string theory interpretation of this solution has been proposed in [12] (see also [29]). It was argued that the $N=6$ case can be seen as four D7-branes accompanied by an orientifold O7-plane. The latter is obtained by modding out $\operatorname{Mink}_{1,7} \times \mathbb{R}^{2}$ with the $\mathbb{Z}_{2^{-}}$

symmetry $(-)^{F_{L}} \Omega I_{8,9}=-T^{-4}$ and hence it carries -4 units of D7-brane charge. The combination $(-)^{F_{L}} \Omega$ acting on the world-sheet fields of the type IIB superstring has the same effect as a $-\mathbb{1}$ transformation has on the space-time fields of type IIB supergravity. In the orientifold limit, the four D7-branes coincide with the O7-plane and cancel the R-R charge. The remaining monodromy is $-\mathbb{1}$. 

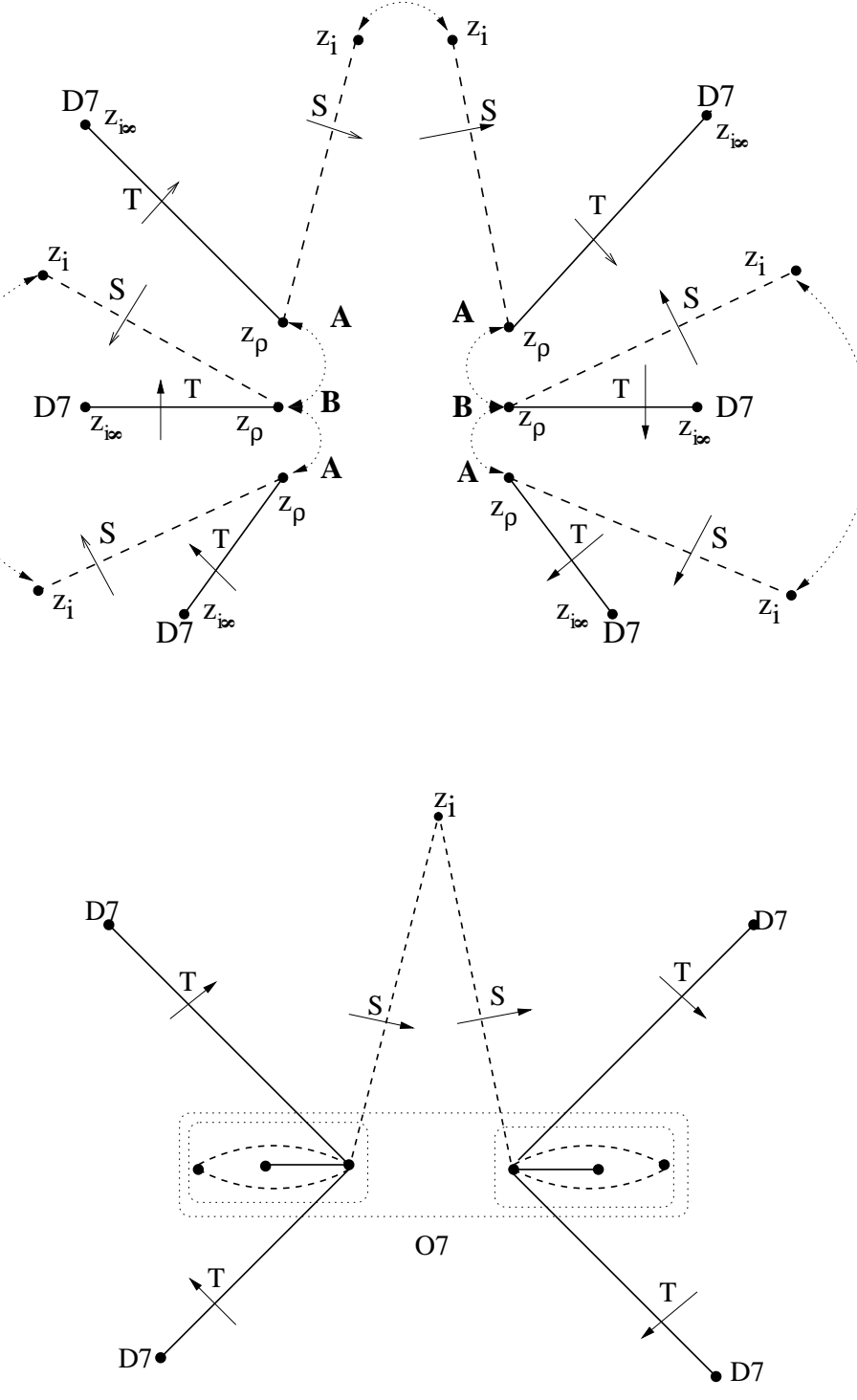

Figure 3: The most general supersymmetric $N=6$ solution (lower diagram) with only non-trivial T-monodromies around the points $z_{i \infty}$, where the D\%-branes are located can be constructed from six elementary $N=1$ solutions (upper diagram) by forcing some branes to coincide. The filled (dashed) lines are $T(S)$ branch cuts while the dotted lines in the upper diagram relate the points which are made coincident to obtain the lower diagram. 
In the $N=6$ solution, the four separate branes with $T$-monodromy in figure 3 are to be interpreted as the D7-branes. In addition, there are two composite objects with monodromy $T^{-2} S^{-1}$ and $S^{-1} T^{-2}$. These monodromies can be written as

$$
M_{1,2} T M_{1,2}^{-1} \quad \text { with } \quad M_{1}= \pm\left(\begin{array}{cc}
1 & \lambda_{1} \\
-1 & 1-\lambda_{1}
\end{array}\right), \quad M_{2}= \pm\left(\begin{array}{cc}
1 & \lambda_{2} \\
1 & 1+\lambda_{2}
\end{array}\right)
$$

respectively. Due to the above relations these branes can be viewed as $S L(2, \mathbb{Z})$-transformed versions of D7-branes. Instead of $\tau \rightarrow i \infty$ the complex scalar goes to the real line in their vicinity, and henceforth IIB perturbative string theory is not valid there. Only the monodromy at infinity has a perturbative meaning. For this reason the two composite objects should be considered as one single object, i.e. the O7-plane. In the perturbative limit this composite object indeed has a monodromy given by $-T^{-4}$. It is clear from the picture that one can take at most four out of the six D7-branes coincident.

The orientifold limit corresponds to taking the most general solution of the equation $j(\tau)=$ cst. For the $N=6$ solution this means that all points $z_{i, \rho, i \infty} \neq \infty$ are coincident at some finite value for $z$. The only remnant is the deficit angle of $\pi$ and the $S L(2, \mathbb{Z})$ transformation $-\mathbb{1}$ around this point. There does not exist a limit in which the O7-plane shrinks to a point while the four D7-branes remain at finite distances from the orientifold point.

\section{The $N=12,24$ solutions with only D7-branes}

The $N=12,24$ solutions can be constructed in more than one way. For example the $N=24$ solution may be constructed by gluing four $N=6$ solutions. This leads to a configuration which at infinity (which in this case is a perturbative region) seems to be composed out of four groups of O7-planes each of which is accompanied by four D7-branes. In the orientifold limit, $\tau$ is an arbitrary constant, this $N=24$ solution is interpreted as modding out Mink $_{1,7} \times T^{2}$, with compact transverse space, with the same $\mathbb{Z}_{2}$-symmetry as before.

It was realized in [28] that apart from the $N=6$ solution and its embedding in the $N=24$ solution one could also consider configurations with $N=24$ which are made by joining solutions whose asymptotic value for $\tau$ is either $i$ or $\rho$. The asymptotic value of $\tau$ for $N=6$ can be both $i$ and $\rho$, for the $N=9$ solution it must be $i$, and for the $N=8,10$ solutions it must be $\rho$. If we take the orbifold limit of the $N=6,8,9,10$ solutions we get the orbifolds points of $T^{4} / \mathbb{Z}_{n}$ for $n=3,4,6$. For example the solution of [28] which in the orbifold limit $\tau=i$ becomes $T^{4} / \mathbb{Z}_{4}$ is made out of two $N=9$ solutions and one $N=6$ solution.

Since the $N=6,12,24$ solutions all have a perturbative asymptotic regime it is interesting to count the number of free parameters in these solutions which correspond to the relative motion of the D7-branes. In subsection 6.2 it was derived that for general $N$ the number of real free parameters is $2(2 N-2)+1$. For $N=6,12,24$ there are a number 


$$
2(N-N / 2+N-N / 3)
$$

more real parameters as compared to the situation in which all point $z_{i}$ are grouped in doublets and all points $z_{\rho}$ are grouped in triplets. For example $N-N / 3$ is the number of parameters one has to fix to go from a configuration in which non of the points $z_{\rho}$ are coincident to a configuration in which all points $z_{\rho}$ are organized in triplets. We count

$$
4 N-3-2(N-N / 2+N-N / 3)=5 N / 3-3
$$

free real parameters. Thus for the $N=6,12,24 \mathrm{~F}$-theory solutions there is one real parameter plus $3,8,18$ complex parameters, respectively.

It has been argued in [10] that the $N=24$ configuration consisting only of D7-branes can be interpreted as an 8-dimensional supergravity coupled to 19 vector multiplets obtained via a $K 3$ compactification of $\mathrm{F}$-theory ${ }^{17}$. In the orientifold limit this theory has been argued to be dual to heterotic on a two-torus [10,29].

\subsection{A solution with monodromy group $\Gamma_{0}(2)$}

Here we present an explicit realization of a solution whose monodromy group is $\Gamma_{0}(2)$. We take for $\tau$ and $f$ the following:

$$
\begin{aligned}
j_{\Gamma_{0}(2)}(\tau) & =\frac{1}{(1+i)^{12}}\left(\frac{\eta(\tau)}{\eta(2 \tau)}\right)^{24}=\frac{z-z_{0}}{z-z_{i \infty}}, \\
f & =\eta(\tau) \eta(2 \tau)\left(z-z_{0}\right)^{-3 / 24}\left(z-z_{i \infty}\right)^{-3 / 24} .
\end{aligned}
$$

This solution consists of one D7-brane $(p=1)$ and the S-dual of a D7-brane with $T^{2}$ monodromy and no $\operatorname{det} Q>0$-branes. The monodromy of $\tau$ for this S-dual D7-brane is $S T^{2} S(q=2)$. Within the subgroup $\Gamma_{0}(2)$ these two branes are inequivalent.

This simple example is included to show explicitly that one can construct globally welldefined supersymmetric solutions with finite energy without using the full $S L(2, \mathbb{Z})$ duality group of type IIB and that a priori the monodromy group of a 7-brane configuration need not equal the full duality group. It it clear that there is a wealth of other possible choices, leading to different supersymmetric configurations of 7-branes. Here we will not pursue this point any further.

\footnotetext{
${ }^{17}$ Of these 19 vector multiplets 18 are to attributed the presence of the 24 D7-branes (see equation (6.16)) and one comes from the reduction of the IIB supergravity (see footnote 6). Note that there is an additional graviphoton sitting in the supergravity multiplet leading to a total of 20 vectors.
} 


\section{Conclusions}

In this paper we have re-derived old results and found new possibilities for globally welldefined, supersymmetric 7-brane solutions of finite energy in type IIB supergravity. A number of things are worth pointing out.

One of our findings is that the most general supersymmetric solutions contain objects with $\operatorname{det} Q$ both equal to zero and positive. By considering particular configurations of the $N=6,12,24$ solutions the results of [1] were reproduced, using a different and arguably more explicit argument. In this paper the global properties of the Killing spinor have played a key role, whereas [1] focussed on the uplift to a manifold of special holonomy.

It is instructive to consider why we have found more supersymmetric 7-brane configurations. The defining property of the configurations of [1] is that they all lead to smooth CY 2-folds in the $(z, \tau)$ directions. The distinguishing feature of the new supersymmetric 7-brane configurations we constructed in this paper is that they do not correspond to a CY 2 -fold reduction of F-theory. This is due to the presence of the $\operatorname{det} Q>0$-branes which lead to conical singularities at the points $z_{i}$ and $z_{\rho}$ that cannot be resolved into a Ricci-flat Kähler manifold. It would, however, still be a manifold of $S U(2)$ holonomy.

The status and role of the $\operatorname{det} Q>0$-branes in string theory is at present unclear. One can adopt a number of different viewpoints. For example, an interpretation as bound states of D7-branes and $S L(2, \mathbb{Z})$ transforms thereof can be put forward since any det $Q>$ 0 monodromy can be written as the product of $\operatorname{det} Q=0$ monodromies. Indeed, the approximate solutions (3.25), (3.24) around the points $z_{\rho}$ and $z_{i}$ can be written in terms of a distribution of D7-branes [36]. Similar bound states occurred when we discussed solutions with $\Gamma_{0}(2)$ monodromy group in subsection 6.4 .

Another interpretation is in terms of O7-planes, as has been discussed in section 6.3. The results of the present paper are more in line with the latter point of view. The D7-brane necessarily comes with two additional points $z_{i}$ and $z_{\rho}$, to cancel its mass and $S L(2, \mathbb{Z})$-charge, and thus to allow for a globally well-defined solution. This is reminiscent of the D8-brane, which also is necessarily paired with an O8-plane, see e.g. [33,34]. The difference is that the D8-brane carries Abelian charge, which is exactly cancelled by the O8-plane, while the D7-brane requires points of $S L(2, \mathbb{Z})$ monodromy. Of course, for $p$ branes with $p \leq 6$ these complications do not occur since their transverse space is at least 3-dimensional, and hence allows for a net charge.

It turns out that there is an intriguing relation between the approximate $D=107$-brane solutions (3.24)-(3.25), which are expansions of modular functions around the orbifold points of the $\tau$-plane, and domain walls in $D=9$ dimensions. In fact, the approximate 7-brane solutions for det $Q>0$ were first found by uplifting domain walls of $D=9$ gauged maximal supergravity with gauge group $S O(2)$ [24]. The reason for this relationship is that the approximate 7-brane solutions, in contrast to the full solution, have an isometry in their

transverse space. More precisely, their angular dependence (in the transverse space) is of the form of an $S L(2, \mathbb{Z})$ transformation and this allows a twisted Scherk-Schwarz reduction over this direction to $D=9$ dimensions.

In general, the supersymmetric domain walls of gauged maximal supergravities in $D$ 
dimensions give rise to the near-horizon limit of (distributions of) $p$-branes with $D=p+2$ $[35,36]$. For 7-branes, however, there are problems with the definition of the near-horizon limit (due to the absence of a corresponding 'dual' frame). In view of the domain wall connection, it thus seems that the expansions (3.24), (3.25) around the orbifold points should be seen as the analogue of near-horizon limits for 7-branes. Indeed, in this limit there is an $S^{1}$ isometry in the solution, in analogy with the spherical part in the nearhorizon limit of $p$-branes with $p \leq 6$. It would be interesting to pursue these ideas further.

\section{Acknowledgements}

We would like to thank D. Sorokin for useful discussions, in particular, regarding section 2. T.O. and D.R. would like to thank the University of Groningen for hospitality, while J.H. and D.R. would like to thank the Universidad Autónoma in Madrid for hospitality. E.B. and T.O. are supported by the European Commission FP6 program MRTN-CT2004-005104 in which E.B. is associated to Utrecht university and T.O. is associated to the IFT-UAM/CSIC in Madrid. The work of E.B. and T.O. is partially supported by the Spanish grant BFM2003-01090. The work of T.O. has been partially supported by the Comunidad de Madrid grant HEPHACOS P-ESP-00346. Part of this work was completed while D.R. was a post-doc at King's College London, for which he would like to acknowledge the PPARC grant PPA/G/O/2002/00475. In addition, he is presently supported by the European EC-RTN project MRTN-CT-2004-005104, MCYT FPA 2004-04582-C02-01 and CIRIT GC 2005SGR-00564. J.H. is supported by a Breedte Strategie grant of the University of Groningen.

\section{References}

[1] B. R. Greene, A. D. Shapere, C. Vafa and S. T. Yau, Nucl. Phys. B 337, 1 (1990).

[2] J. Polchinski, Phys. Rev. Lett. 75, 4724 (1995) [arXiv:hep-th/9510017].

[3] G. W. Gibbons, M. B. Green and M. J. Perry, Phys. Lett. B 370, 37 (1996) [arXiv:hep-th/9511080].

[4] M. Grana and J. Polchinski, Phys. Rev. D 65 (2002) 126005 [arXiv:hep-th/0106014].

[5] A. Karch and A. Katz, Fortsch. Phys. 51, 759 (2003).

[6] I. Kirsch and D. Vaman, Phys. Rev. D 72, 026007 (2005) [arXiv:hep-th/0505164].

[7] K. Dasgupta, C. Herdeiro, S. Hirano and R. Kallosh, Phys. Rev. D 65, 126002 (2002) [arXiv:hep-th/0203019].

[8] S. Kachru, R. Kallosh, A. Linde and S. P. Trivedi, Phys. Rev. D 68, 046005 (2003) [arXiv:hep-th/0301240]. 
[9] C. P. Burgess, R. Kallosh and F. Quevedo, JHEP 0310, 056 (2003) [arXiv:hepth/0309187].

[10] C. Vafa, Nucl. Phys. B 469, 403 (1996) [arXiv:hep-th/9602022].

[11] E. A. Bergshoeff, M. de Roo, S. F. Kerstan, T. Ortín and F. Riccioni, arXiv:hepth/0611036.

[12] A. Sen, Nucl. Phys. B 475, 562 (1996) [arXiv:hep-th/9605150].

[13] J. H. Schwarz and P. C. West, Phys. Lett. B 126 (1983) 301.

[14] J. H. Schwarz, Nucl. Phys. B 226 (1983) 269.

[15] P. S. Howe and P. C. West, Nucl. Phys. B 238 (1984) 181.

[16] J. M. Maldacena, arXiv:hep-th/9607235.

[17] P. Pasti, D. P. Sorokin and M. Tonin, Phys. Rev. D 52, 4277 (1995) [arXiv:hep-th/9506109].

[18] P. Pasti, D. P. Sorokin and M. Tonin, Phys. Rev. D 55, 6292 (1997) [arXiv:hep-th/9611100].

[19] E. Bergshoeff, J. Hartong and D. Sorokin, work in progress.

[20] E. A. Bergshoeff, M. de Roo, S. F. Kerstan, T. Ortín and F. Riccioni, JHEP 0606, 006 (2006) [arXiv:hep-th/0601128].

[21] E. Bergshoeff, C. M. Hull and T. Ortín, Nucl. Phys. B 451, 547 (1995) [arXiv:hep-th/9504081].

[22] P. Meessen and T. Ortín, Nucl. Phys. B 541 (1999) 195 [arXiv:hep-th/9806120].

[23] E. Lozano-Tellechea and T. Ortín, Nucl. Phys. B $\mathbf{6 0 7}$ (2001) 213 [arXiv:hep-th/0012051].

[24] E. Bergshoeff, U. Gran and D. Roest, Class. Quant. Grav. 19 (2002) 4207 [arXiv:hep-th/0203202].

[25] S. Deser, R. Jackiw and G. 't Hooft, Annals Phys. 152, 220 (1984).

[26] T.M. Apostol, Modular Functions and Dirichlet Series in Number Theory, SpringerVerlag, 1976.

[27] B. Schoeneberg, Elliptic Modular Functions Springer-Verlag, 1974.

[28] K. Dasgupta and S. Mukhi, Phys. Lett. B 385, 125 (1996) [arXiv:hep-th/9606044]. 
[29] A. Dabholkar, arXiv:hep-th/9804208.

[30] P. S. Howe, J. M. Izquierdo, G. Papadopoulos and P. K. Townsend, Nucl. Phys. B 467, 183 (1996) [arXiv:hep-th/9505032].

[31] J.-P. Serre, A course in Arithmetic, Springer-Verlag (1978).

[32] P. Meessen and T. Ortín, Nucl. Phys. B 749 (2006) 291 [arXiv:hep-th/0603099].

[33] J. Polchinski and E. Witten, Nucl. Phys. B 460, 525 (1996) [arXiv:hep-th/9510169].

[34] E. Bergshoeff, R. Kallosh, T. Ortín, D. Roest and A. Van Proeyen, Class. Quant. Grav. 18, 3359 (2001) [arXiv:hep-th/0103233].

[35] H. J. Boonstra, K. Skenderis and P. K. Townsend, JHEP 9901 (1999) 003 [arXiv:hepth/9807137].

[36] E. Bergshoeff, M. Nielsen and D. Roest, JHEP 0407 (2004) 006 [arXiv:hepth/0404100]. 